\title{
ASPECTOS SOCIOECONÔMICOS E A COINFECÇÃO TUBERCULOSE/HIV NO BRASIL: UMA REVISÃO DA LITERATURA
}

\author{
Cleizimara Cavalcante Nunes ${ }^{1}$, Randerson José de Araujo Sousa ${ }^{1}$, Ana \\ Gabriela de Sousa Costa1 ${ }^{1}$, Lígia Amaral Filgueiras ${ }^{2}$, Yane Santos Almeida ${ }^{3}$ \\ ${ }^{1}$ Graduandos em Medicina, Universidade do Estado do Pará, Brasil. \\ 2 Doutora em Antropologia, Docente da Universidade do Estado do Pará, Brasil \\ ${ }^{3}$ Mestra em Biotecnologia, Docente da Universidade do Estado do Pará, Brasil \\ Email para correspondência: cleizimaracavalcante@gmail.com
}

\section{Resumo}

\begin{abstract}
Objetivo: analisar como os aspectos socioeconômicos impactam na evolução da coinfecção tuberculose(TB)/Vírus da Imunodeficiência Humana(HIV), no Brasil. Metodologia: revisão integrativa com abordagem descritiva, feita por buscas nas bases de dados BVS e PubMed. Os artigos selecionados corresponderam ao período de 2015 a 2019, nos idiomas inglês, espanhol e português. Foram estabelecidos critérios de exclusão e inclusão. A organização das pesquisas foi realizada com auxílio do software Microsoft Excel® 2010, onde se coletaram os seguintes dados: título, autores, ano, delineamento, amostra, principais resultados e considerações. Resultados: da totalidade de 116 artigos, nove foram adequados. Observou-se a prevalência de pacientes inseridos em vulnerabilidades sociais, destacando-se baixa escolaridade, morador de rua, profissão exercida e baixa renda. Ademais, evidenciaram-se indivíduos do sexo masculino, negros e usuários de álcool e drogas. Constatou-se a demora no diagnóstico da coinfecção e desfechos desfavoráveis e, que fatores socioeconômicos impactam o estado de coinfecção TB/HIV e a evolução dessa associação. Conclusão: a vulnerabilidade, a ineficácia no diagnóstico, falta de amparo profissional, social e familiar contribuem com a evolução negativa da coinfecção e com o problema de saúde, que é ainda sustentado pela carência de políticas públicas eficazes que considerem os diversos aspectos que contribuem para a prevalência dessas doenças.
\end{abstract}

Palavras-chave: preconceito, saúde pública, vulnerabilidade social, políticas públicas.

\begin{abstract}
Objective: to analyze how socioeconomic aspects impact the evolution of tuberculosis (TB)/Human Immunodeficiency Virus (HIV) coinfection in Brazil. Methodology: this was an integrative review with a descriptive approach, carried out by searching the BVS and PubMed databases. The selected articles corresponded to the period from 2015 to 2019, in English, Spanish and Portuguese. Exclusion and inclusion criteria were established. The organization of the research was carried out with the aid of Microsoft Excel® 2010 software, where
\end{abstract}




\begin{abstract}
the following data were collected: title, authors, year, design, sample, main results and considerations. Results: of the total of 116 articles, nine were adequate. It was observed the prevalence of patients inserted in social vulnerabilities, highlighting low education, homeless person, profession and low income. In addition, male, black and alcohol and drug users were found. There was a delay in the diagnosis of coinfection and unfavorable outcomes, and that socioeconomic factors impact the state of TB / HIV coinfection and the evolution of this association. Conclusion: vulnerability, ineffective diagnosis, lack of professional, social and family support contribute to the negative evolution of co-infection and to the health problem, which is still supported by the lack of effective public policies that consider the different aspects that contribute to the prevalence of these diseases.
\end{abstract}

Keywords: prejudice, public health, social vulnerability, public policy.

\title{
1 Introdução
}

A tuberculose (TB) é uma doença infecciosa causada pela bactéria Mycobacterium tuberculosis que atinge os pulmões e outros locais do corpo, caracterizando a tuberculose pulmonar e extrapulmonar, respectivamente (NOGUEIRA et al., 2012). Após o contato, a infecção pode permanecer em latência por anos, sendo que esse tempo varia de acordo com o estado imunológico do infectado (SANTOS et al., 2017).

O bacilo de Koch ainda é um problema de saúde em vários países, tendo o Brasil um destaque significativo, pois anualmente 69 mil novos casos surgem e cerca de 4.500 óbitos são resultantes da tuberculose, além disso, quando se trata de uma visão global fica mais evidente a gravidade da persistência dessa enfermidade, pois em 2015 o total de vítimas foi de 1,8 milhões nos diferentes gêneros e faixas etárias (BRASIL, 2017a).

Ao passo, estima-se que um terço das quase 35 milhões de pessoas, em todo o mundo, portadoras do Vírus da Imunodeficiência Humana (HIV), também está com Mycobacterium tuberculosis, o que os torna pacientes potenciais para o desenvolvimento da tuberculose ativa, bem como para o aparecimento de agravantes clínicos, aumento das internações, além da elevação no índice de evasão ao tratamento e, consequentemente, maior número de óbitos (MAGNABOSCO et al., 2019).

Considera-se que o HIV, desde 1980, seja um fator preponderante no reaparecimento da tuberculose nos mais diferentes países, sendo que esse vírus alterou a história natural da forma ativa da tuberculose e promoveu modificações expressivas, sobretudo, na epidemiologia e na clínica da bactéria. Além do mais, pessoas portadoras do HIV possuem, anualmente, um risco de 
reativar a latência da infecção por tuberculose, aproximadamente, entre 3 e $15 \%$ em comparação com 0,01 a $0,1 \%$ para a população em geral (PRADO et al., 2014).

Essas patologias ainda são bastante negligenciadas, principalmente, por sua associação com as vulnerabilidades sociais dos indivíduos, como a pobreza, o que corrobora para com as desigualdades na saúde (BRAND et al., 2019). Nesse sentido, ao se tratar da coinfecção TB/HIV, o Brasil, aparece entre os 20 países do mundo com a maior incidência, sinalizando 13 casos por 100.000 habitantes, porém os locais mais atingidos se encontram no continente africano, 72 casos por 100.000 habitantes (WHO, 2017).

A coinfecção TB/HIV, apresenta-se para a saúde pública nos últimos anos, como um desafio significativo, uma vez que, a tuberculose é o principal fator de risco para óbitos em indivíduos com a Síndrome da Imunodeficiência Adquirida - três óbitos para cada dez pessoas -, sendo geralmente letal em pacientes infectados pelo HIV (BRUNELLO et al., 2011).

Dessa forma, em face dos determinantes sociais que contribuem para o processamento desse cenário, este estudo objetivou realizar um levantamento bibliográfico, a fim de analisar como os aspectos socioeconômicos impactam na evolução da coinfecção tuberculose - HIV no Brasil, no período de 2015 a 2019, em face da prevalência dessa combinação de infecções no país.

\section{Metodologia}

Trata-se de uma revisão da literatura com formato integrativo em que o levantamento bibliográfico consistiu ao período de 2015 a 2019. Esse tipo de estudo apresenta expressivo destaque quando diz respeito às pesquisas em saúde, sendo um método de análise importante de publicações científicas ao sintetizar os estudos sobre um determinado tema e possibilitar a identificação de lacunas no conhecimento existente para instigar novas pesquisas originais (COSTA et al., 2020).

No levantamento bibliográfico, seguiram-se as seis etapas para a construção de uma revisão propostas por SOUZA et al. (2010). Nesse sentido, estabeleceu-se, primeiramente, a pergunta que conduziu a busca. Em seguida, para realizar a catalogação dos artigos foram adotadas as respectivas bases 
de dados: Biblioteca Virtual em Saúde (BVS) e PubMed, bem como, foram utilizados os seguintes descritores em português, inglês e espanhol: "tuberculose", "Brasil", "HIV", "classe social", ("social class" OR "classe social" OR "clase social") AND (tuberculosis OR tuberculose) AND HIV AND (Brasil or Brazil).

Após isso, o próximo passo consistiu na seleção e na análise crítica dos artigos, o qual cumpriu as seguintes etapas: 1) foi feita a leitura breve do título e do resumo; 2) aplicaram-se os critérios de inclusão: artigos científicos publicados em língua portuguesa, inglesa e espanhola, estudos científicos disponíveis integralmente, artigos produzidos com a realidade brasileira, além dos de exclusão: teses, dissertações, trabalhos de conclusão de curso, artigos de revisão e artigos que não tangenciavam a temática proposta; 3) quando dentro da temática abordada, foi feita a leitura na íntegra dos artigos e a seleção das informações importantes à revisão.

Posteriormente, as informações extraídas dos artigos selecionados foram sintetizadas em um quadro, o qual sua estruturação foi auxiliada pelo software Microsoft Exce/® 2010, onde todos os trabalhos analisados e que se encaixaram na temática estabelecida foram organizados por: título, autores, ano, delineamento, amostra, principais resultados e considerações. Por fim, os resultados foram descritos, discutidos e apresentados de forma clara e completa, sendo as lacunas e as inferências sinalizadas, quando pertinentes. Todas as etapas, portanto, foram cumpridas, bem como, os aspectos éticos que nortearam o processo de elaboração do artigo.

\section{Resultados e discussão}

Foi selecionado um total de 116 artigos utilizando os descritores citados. Desses, somente nove artigos se adequaram à temática desta revisão e foram sintetizados no Quadro 1. Ademais, no recorte temporal estabelecido, o ano que concentrou maiores achados foi o de 2016, com três artigos e todos os anos apresentaram pelo menos um estudo, nas plataformas, sob os critérios estabelecidos.

O estado de coinfecção representa um agravo para o paciente com HIV/Aids, pois além de ocasionar transformações indesejadas, como 0 
aumento da quantidade de medicamentos e possíveis efeitos adversos mais intensos, bem como um crescente risco de óbito (BRASIL, 2017a), esses pacientes apresentam probabilidade muito mais elevada em contrair Mycobacterium tuberculosis e desenvolver a doença em relação ao restante da população, o que pode resultar em menores chances de cura (MAGNABOSCO et al., 2019). Desse modo, múltiplos fatores incidem sobre o quadro de coinfecção TB/HIV e atuam como agravantes e, por vezes, como causalidade.

Nesse viés e no que tange o perfil dos mais vulneráveis, os critérios de raça se mostraram significativos tanto ao se observar a incidência da coinfecção quanto na efetividade do tratamento, fato evidenciado por Escada et al. (2017), ao identificarem os números díspares de morbimortalidade de pacientes negros, com o dobro de mortalidade, em relação aos brancos, e por Prado et al. (2017), ao verificarem maior índice de mortes devido à coinfecção entre negros.

No entanto, as desigualdades analisadas nos estudos também podem ser percebidas além do campo da TB/HIV e são, na realidade, o reflexo de uma problemática bem mais ampla, que é a forma como o racismo cultural e institucional se estende e se processa na assistência à saúde (WILLIAMS \& PRIEST, 2015), mesmo com a existência da Política Nacional de Saúde Integral da População Negra, a qual objetiva a promoção integral de saúde à população negra, na tentativa de reduzir as desigualdades étnico-raciais, bem como combater o racismo e à discriminação nas esferas do Sistema Único de Saúde (BATISTA \& BARROS, 2017).

Outro fator que possui estreita relação com as doenças, segundo Damásio et al. (2016), é o gênero dos indivíduos, uma vez que, o sexo masculino prevalece entre os infectados, sendo a principal via para a infecção por HIV a sexual. Concomitantemente, Neves et al. (2018), associaram a alta prevalência entre homens ao seu estilo de vida, marcado, comumente pela exposição aos agentes infecciosos, além de serem mais propensos a resistir ao tratamento.

Essas condições que permeiam o gênero masculino recaem e se relacionam à saúde do homem, aos estereótipos de gênero e preceitos sexistas (DANTAS \& COUTO, 2018), conceitos que culminam em um quadro de fragilidade devido aos estigmas sociais, que estimulam a adoção de um 
comportamento de risco, interferindo na forma como o homem lida tanto com questões de saúde, quanto com a própria integridade física (MIRANDA et al., 2018).

Quadro 1: Síntese dos artigos acerca da coinfecção Tuberculose/HIV associada aos aspectos socioeconômicos, no Brasil, de 2015 a 2019.

\begin{tabular}{|c|c|c|c|}
\hline $\begin{array}{c}\text { Título } \\
\text { Autores/ Ano }\end{array}$ & $\begin{array}{c}\text { Delineamento } \\
\text { Amostra }\end{array}$ & $\begin{array}{l}\text { Principais } \\
\text { resultados }\end{array}$ & Considerações \\
\hline $\begin{array}{c}\text { Clinical and } \\
\text { epidemiological } \\
\text { characteristics } \\
\text { associated with } \\
\text { unfavorable } \\
\text { tuberculosis } \\
\text { treatment outcomes } \\
\text { in TB-HIV co- } \\
\text { infected patients in } \\
\text { Brazil: a hierarchical } \\
\text { polytomous analysis } \\
\text { PRADO et al. (2017) }\end{array}$ & $\begin{array}{c}\text { Transversal } \\
68.295\end{array}$ & $\begin{array}{c}\text { A probabilidade de } \\
\text { morte, em } \\
\text { comparação a } \\
\text { outros pacientes } \\
\text { com tuberculose, é } \\
\text { maior entre } \\
\text { indivíduos } \\
\text { diagnosticados } \\
\text { com HIV e com } \\
\text { problemas com } \\
\text { álcool. Além disso, } \\
\text { pessoas com } \\
\text { HIV/TB, que } \\
\text { morreram por } \\
\text { tuberculose, são } \\
\text { em maioria negros } \\
\text { e mais idosas. }\end{array}$ & $\begin{array}{c}\text { Identificou que } \\
\text { características } \\
\text { socioeconômicas, } \\
\text { como raça e } \\
\text { educação, em } \\
\text { casos de } \\
\text { coinfecção por } \\
\text { HIV exercem } \\
\text { influência sobre o } \\
\text { tratamento da } \\
\text { doença, devido à } \\
\text { vulnerabilidade } \\
\text { dos indivíduos e } \\
\text { falta de } \\
\text { assistência. }\end{array}$ \\
\hline $\begin{array}{c}\text { Coinfecção } \\
\text { tuberculose/HIV/aids } \\
\text { em Porto Alegre, RS } \\
\text { - invisibilidade e } \\
\text { silenciamento dos } \\
\text { grupos mais } \\
\text { afetados } \\
\text { ROSSETTO et al. } \\
\text { (2019) }\end{array}$ & $\begin{array}{l}\text { Qualitativo } \\
20\end{array}$ & $\begin{array}{c}\text { O desenvolvimento } \\
\text { de tuberculose/HIV } \\
\text { entre os } \\
\text { entrevistados } \\
\text { ocorreu sob } \\
\text { circunstâncias de } \\
\text { vulnerabilidade } \\
\text { social, } \\
\text { determinadas por } \\
\text { classe, gênero, } \\
\text { raça e profissão, } \\
\text { com destaque para } \\
\text { profissionais do } \\
\text { sexo, que se } \\
\text { associaram a um } \\
\text { cenário de omissão } \\
\text { e negligência por } \\
\text { parte de } \\
\text { profissionais da } \\
\text { saúde. }\end{array}$ & $\begin{array}{c}\text { O } \\
\text { desfavorecimento } \\
\text { social foi } \\
\text { determinante } \\
\text { para o processo } \\
\text { de invisibilização } \\
\text { dos pacientes } \\
\text { com } \\
\text { tuberculose/HIV, } \\
\text { influindo tanto na } \\
\text { demora para a } \\
\text { identificação da } \\
\text { coinfecção } \\
\text { quanto na } \\
\text { eficácia do } \\
\text { tratamento. }\end{array}$ \\
\hline
\end{tabular}




\begin{tabular}{|c|c|c|c|}
\hline $\begin{array}{l}\text { Fatores sociais, } \\
\text { clínicos e de adesão } \\
\text { em coinfectados por } \\
\text { HIV/Tuberculose: } \\
\text { estudo descritivo } \\
\text { DAMÁSIO et al. } \\
(2016)\end{array}$ & $\begin{array}{l}\text { Descritivo } \\
34\end{array}$ & $\begin{array}{l}\text { Boa parte dos } \\
\text { entrevistados } \\
\text { erado sexo } \\
\text { masculino e } \\
\text { apresentava baixa } \\
\text { renda e baixa } \\
\text { escolaridade. O } \\
\text { estudo identificou } \\
\text { que quanto maior a } \\
\text { demora para } \\
\text { diagnosticar a } \\
\text { coinfecção, maior a } \\
\text { dificuldade para } \\
\text { adesão ao } \\
\text { tratamento. }\end{array}$ & $\begin{array}{c}\text { É necessária a } \\
\text { implementação } \\
\text { de estratégias de } \\
\text { adesão ao } \\
\text { tratamento por } \\
\text { parte dos } \\
\text { profissionais de } \\
\text { saúde, em vista } \\
\text { às dificuldades } \\
\text { enfrentadas pelos } \\
\text { pacientes ao } \\
\text { longo da terapia } \\
\text { medicamentosa. }\end{array}$ \\
\hline $\begin{array}{c}\text { Identifying } \\
\text { socioeconomic, } \\
\text { epidemiological and } \\
\text { operational } \\
\text { scenarios for } \\
\text { tuberculosis control } \\
\text { in Brazil: an } \\
\text { ecological study } \\
\text { PELISSARI et al. } \\
\text { (2018) }\end{array}$ & $\begin{array}{l}\text { Descritivo } \\
\text { analítico } \\
67.777\end{array}$ & $\begin{array}{l}\text { O estudo sugere } \\
\text { subdetecção de } \\
\text { HIV entre } \\
\text { indivíduos com } \\
\text { tuberculose, devido } \\
\text { à carência de } \\
\text { testagem. Assim } \\
\text { como indica a } \\
\text { associação entre } \\
\text { tuberculose e } \\
\text { indivíduos em } \\
\text { situação de } \\
\text { vulnerabilidade. }\end{array}$ & $\begin{array}{c}\text { As disparidades } \\
\text { socioeconômicas } \\
\text { brasileiras atuam } \\
\text { como um } \\
\text { empecilho para o } \\
\text { tratamento de } \\
\text { tuberculose, } \\
\text { sendo imperativa } \\
\text { a adoção de } \\
\text { medidas de } \\
\text { monitorização do } \\
\text { quadro real da } \\
\text { doença no Brasil, } \\
\text { principalmente } \\
\text { entre as camadas } \\
\text { marginalizadas. }\end{array}$ \\
\hline $\begin{array}{l}\text { Mortality in patients } \\
\text { with HIV-1 and } \\
\text { tuberculosis co- } \\
\text { infection in Rio de } \\
\text { Janeiro, Brazil - } \\
\text { associated factors } \\
\text { and causes of death } \\
\text { ESCADA et al. } \\
\text { (2017) }\end{array}$ & $\begin{array}{c}\text { Observacional } \\
\text { retrospectivo } \\
310\end{array}$ & $\begin{array}{l}\text { É ressaltada a alta } \\
\text { mortalidade e } \\
\text { morbidade entre } \\
\text { indivíduos com } \\
\text { tuberculose e HIV, } \\
\text { sendo que o atraso } \\
\text { do diagnóstico da } \\
\text { coinfecção é um } \\
\text { fator que aumenta } \\
\text { a mortalidade. } \\
\text { Pacientes negros } \\
\text { apresentaram o } \\
\text { dobro da } \\
\text { mortalidade em } \\
\text { comparação aos } \\
\text { pacientes brancos. }\end{array}$ & $\begin{array}{l}\text { Para prover } \\
\text { saúde de forma } \\
\text { igualitária, é } \\
\text { necessário } \\
\text { resolver as } \\
\text { questões de } \\
\text { desigualdades } \\
\text { sociais no acesso } \\
\text { ao tratamento } \\
\text { eficaz, de forma a } \\
\text { reduzir a } \\
\text { mortalidade. }\end{array}$ \\
\hline $\begin{array}{l}\text { Os significados da } \\
\text { comorbidade para }\end{array}$ & $\begin{array}{c}\text { Descritivo } \\
10\end{array}$ & $\begin{array}{l}\text { A maior parte dos } \\
\text { entrevistados } \\
\text { possui baixa }\end{array}$ & $\begin{array}{l}\text { A coinfecção por } \\
\text { tuberculose e HIV } \\
\text { imprime muitas }\end{array}$ \\
\hline
\end{tabular}




\begin{tabular}{|c|c|c|c|}
\hline $\begin{array}{l}\text { os pacientes } \\
\text { vivendo com } \\
\text { TB/HIV: } \\
\text { repercussões no } \\
\text { tratamento } \\
\text { SILVA et al. (2015) }\end{array}$ & & $\begin{array}{c}\text { escolaridade e } \\
\text { baixa renda. Foram } \\
\text { relatados } \\
\text { sentimentos de } \\
\text { negação ao } \\
\text { receberem o } \\
\text { diagnóstico de } \\
\text { tuberculose/HIV, } \\
\text { relacionados ao } \\
\text { medo quanto à } \\
\text { concepção da } \\
\text { sociedade a } \\
\text { respeito da } \\
\text { doença. É } \\
\text { ressaltada a } \\
\text { importância do } \\
\text { apoio familiar, } \\
\text { religioso e } \\
\text { acompanhamento } \\
\text { adequado por } \\
\text { profissionais de } \\
\text { saúde. }\end{array}$ & $\begin{array}{l}\text { repercussões } \\
\text { psicossociais, } \\
\text { nesse sentido, o } \\
\text { atendimento } \\
\text { individualizado é } \\
\text { relevante para } \\
\text { que o paciente se } \\
\text { sinta aceito e } \\
\text { siga o } \\
\text { tratamento. }\end{array}$ \\
\hline $\begin{array}{c}\text { Suporte social e } \\
\text { qualidade de vida } \\
\text { de indivíduos com } \\
\text { coinfecção } \\
\text { tuberculose/HIV } \\
\text { NEVES et al. (2018) }\end{array}$ & $\begin{array}{c}\text { Transversal } \\
57\end{array}$ & $\begin{array}{c}\text { Os indivíduos do } \\
\text { estudo apresentam } \\
\text { baixa escolaridade } \\
\text { e renda, sendo a } \\
\text { maioria } \\
\text { heterossexual e do } \\
\text { sexo masculino. A } \\
\text { baixa aceitação } \\
\text { social oriunda da } \\
\text { coinfecção por } \\
\text { tuberculose e HIV } \\
\text { implicou em } \\
\text { redução da } \\
\text { qualidade de vida. }\end{array}$ & $\begin{array}{l}\text { O apoio familiar e } \\
\text { um bom auxílio } \\
\text { profissional } \\
\text { exercem grande } \\
\text { influência quanto } \\
\text { à aceitação da } \\
\text { doença e adesão } \\
\text { ao tratamento. }\end{array}$ \\
\hline $\begin{array}{l}\text { The impact of being } \\
\text { homeless on the } \\
\text { unsuccessful } \\
\text { outcome of } \\
\text { treatment of } \\
\text { pulmonary TB in } \\
\text { São Paulo State, } \\
\text { Brazil } \\
\text { RANZANI et al. } \\
\text { (2016) }\end{array}$ & $\begin{array}{c}\text { Coorte } \\
\text { retrospectiva } \\
61.817\end{array}$ & $\begin{array}{c}\text { Pessoas em } \\
\text { situação de rua } \\
\text { com tuberculose } \\
\text { enfrentam maior } \\
\text { subnotificação } \\
\text { quanto a HIV e } \\
\text { maiores índices da } \\
\text { doença em } \\
\text { comparação à } \\
\text { população geral. A } \\
\text { maior parte } \\
\text { constitui-se por } \\
\text { homens negros e } \\
\text { com baixa }\end{array}$ & $\begin{array}{l}\text { A situação de rua } \\
\text { associada a } \\
\text { outros fatores de } \\
\text { risco, como uso } \\
\text { de drogas e } \\
\text { comorbidades, é } \\
\text { um elemento que } \\
\text { predispõe a } \\
\text { redução da } \\
\text { eficácia do } \\
\text { tratamento para } \\
\text { tuberculose } \\
\text { associada ao } \\
\text { HIV. }\end{array}$ \\
\hline
\end{tabular}




\begin{tabular}{|c|c|c|c|}
\hline & escolaridade. & \\
\hline $\begin{array}{c}\text { Vulnerabilidade e } \\
\text { direitos humanos na } \\
\text { compreensão de } \\
\text { trajetórias de } \\
\text { internação por } \\
\text { tuberculose }\end{array}$ & $\begin{array}{c}\text { Os preconceitos } \\
\text { ainda existentes } \\
\text { principalmente } \\
\text { quanto a AIDS, } \\
\text { presentes } \\
\text { também entre } \\
\text { MAFFACCIOLLI et } \\
\text { al. (2017) }\end{array}$ & $\begin{array}{c}\text { A coinfecção } \\
\text { tuberculose/HIV, } \\
\text { associada ao uso } \\
\text { de álcool e drogas } \\
\text { profissionais de } \\
\text { saúncia em } \\
\text { situação de rua, } \\
\text { consiste em um } \\
\text { fator exacerbador } \\
\text { da vulnerabilidade } \\
\text { social. }\end{array}$ & $\begin{array}{c}\text { contribuem para } \\
\text { o aumento da } \\
\text { marginalização e } \\
\text { dificultam o } \\
\text { acesso ao } \\
\text { tratamento dos } \\
\text { indivíduos com } \\
\text { tuberculose e } \\
\text { HIV. }\end{array}$ \\
\hline
\end{tabular}

Fonte: elaborado pelos autores.

Ao passo, a representatividade social ocupada pelo coinfectado, também pode impactar na assistência, uma vez que, profissões estigmatizadas, como profissional do sexo ou outras atividades repudiadas pela sociedade, bem como a condição de encarcerado, apresentam aspectos cruciais no agravo à saúde, pois são situações em que a baixa escolaridade e qualificação, condição social ainda bastante presente na sociedade brasileira, permitem a submissão a condições insalubres para adquirir o sustento (ROSSETTO et al., 2019).

Essa realidade de nível escolar e de renda diminuídas, foi constatada em grande maioria dos participantes de estudos realizados no Rio de Janeiro e em Ribeirão Preto (SILVA et al, 2015; NEVES et al; 2018), o que sinalizou o quanto as condições socioeconômicas dos indivíduos são capazes de moldar seu comportamento e atitudes, deixando-os mais propensos aos diversos desfechos com a saúde.

Nesse sentido, Pelissari et al. (2018), reforçaram a presença de elevada taxa de incidência da tuberculose em indivíduos em situação de desemprego e de aglomeração, condições características de classes sociais mais pobres e do sistema prisional, mas afirmaram haver ainda um cenário de subnotificação da coinfecção, dada à investigação insuficiente, demonstrada pela baixa quantidade de testes. Sob essa ótica, um estudo no estado do Piauí referiu que 
apesar da indicação da investigação de HIV ser necessária a todos os doentes de tuberculose, a realidade é distinta, pois a oferta de serviços é desigual nas regiões brasileiras (OLIVEIRA et al., 2018).

Em consonância com as disparidades no tratamento ocasionadas por aspectos socioeconômicos, Ranzani et al. (2016), contemplaram outra questão social ao colocarem que estar em situação de rua traz prejuízos nos mais diversos âmbitos, inclusive na questão do diagnóstico de doenças, pois o alcance desses indivíduos aos serviços de saúde é diferente, em comparação àqueles que não estão nessa condição, sendo, um exemplo, a baixa testagem para o HIV, mesmo havendo a alta incidência entre os moradores de rua.

Contudo, além da falta de estratégias políticas, esse quadro também é sustentado pela forma como a sociedade delibera a respeito da realidade desses indivíduos, rotulando-os como carreadores de doenças, viciados e pobres, normalizando o cenário de vulnerabilidade e visualizando problemas de saúde como parte do cotidiano dessa parcela (MATOS et al., 2017). Por isso, generalizar a abordagem quanto aos infectados e aos possíveis potenciais é uma estratégia eficaz de combate tanto à coinfecção quanto ao preconceito presente nas ações de saúde.

É preciso considerar, todavia, que nessa parcela vulnerável da população o uso mais acentuado de drogas e de álcool foi verificado e, esse comportamento implica diretamente na qualidade do tratamento da coinfecção, uma vez que a rotina instável, com horários inespecíficos para dormir e alimentar-se, associado à carência nutricional, configuram-se como agravantes na efetividade terapêutica (RANZANI et al., 2016).

Nessa perspectiva, Maffacciolli et al. (2017), corroboraram ao evidenciarem que o uso de drogas e a condição de morador de rua também são agravantes ao estado de coinfecção e seu processo de acompanhamento em saúde. Em contraponto, as razões por trás do impacto das drogas sobre 0 tratamento não se encontram somente em questões fisiológicas e comportamentais, mas também no preconceito presente entre profissionais de saúde, já que o mesmo estigma quanto às drogas, presente na sociedade, é igualmente reproduzido entre o meio médico (RODRIGUES; DE LIMA; HOLANDA, 2018). 
Ademais, percebe-se que a vulnerabilidade sustentada, a qual muitos sujeitos estão expostos, contribui para o cenário da coinfecção TB/HIV e escancara uma assistência em saúde heterogênea, onde a classe social ocupada, a cor/raça, com destaque para a negra, além da atividade remunerada exercida pelo enfermo, configuram fatores que interferem na prática da saúde e, Rossetto et al. (2019), defendem esse posicionamento ao afirmarem que a postura dos profissionais da área sofre interferências a depender das características socioeconômicas dos pacientes, inviabilizando uma comunicação efetiva e comprometendo a adesão ao tratamento.

A postura de negligência entra, portanto, em desacordo com a tentativa atual de implementação do Plano Nacional pelo Fim da Tuberculose, que determina o maior acesso ao diagnóstico de tuberculose, principalmente, entre indivíduos com HIV e pessoas privadas de liberdade (BRASIL, 2017b). Em 2016, uma determinação similar de prioridade para realização de testes entre a população mais vulnerável, já estava presente no Manual Técnico para diagnóstico da Infecção pelo HIV em adultos e crianças, porém mesmo diante de tais medidas, o Boletim Epidemiológico a respeito da TB/HIV de 2017, identificou que $23,7 \%$ dos casos de TB diagnosticados eram desconhecidos para HIV, sendo que metade dos casos de coinfecção diagnosticados não se encontrava em tratamento (BRASIL, 2017a).

Outra problemática é o processo de aceitação da coinfecção por TB/HIV, podendo ser um impasse para o coinfectado, por se tratarem de doenças permeadas por tabus, impactando em diversos aspectos da vida por meio de mudanças físicas, psicossociais e mesmo espirituais, uma vez que a percepção de si e de crenças pessoais podem ser sensibilizadas e alteradas pelas circunstâncias vivenciadas (SILVA et al, 2015) e Neves et al. (2018), complementam afirmando ser o suporte social, uma ferramenta que impacta positivamente na qualidade de vida dos pacientes, dando ênfase para o apoio de terceiros, atuando, inclusive, como facilitador de aceitação ao tratamento.

Assim, encontrar amparo espiritual e dos profissionais de saúde desde o momento do diagnóstico, além de reduzir os embates supracitados, contribui para a adesão ao tratamento, sendo primordial diante de uma terapêutica prolongada, com efeitos colaterais e grande quantidade de medicamentos, além do mais a família é fundamental na não desistência da terapêutica 
tuberculínica e refúgio para o paciente relatar seus medos e angústias (SILVA et al., 2015; SILVA et al., 2017).

Portanto, estar doente transcende 0 oferecimento de terapia medicamentosa e é indispensável que haja uma percepção do enfermo além da técnica, abrindo espaço para uma escuta compreensiva e que considere, sem julgamentos, todos os aspectos sociais e econômicos que incidem sobre 0 paciente e impactam de forma significativa no processamento da relação médico-paciente e na evolução da doença.

\section{Conclusão}

Observou-se que o perfil socioeconômico possui grande relação com o estado de coinfecção TB/HIV, bem como com a evolução dessa associação de doenças. Nesse contexto, os achados demonstraram maior acometimento em indivíduos com as características: negro, de baixa escolaridade e renda, do gênero masculino, trabalhadores com atividades estigmatizadas, moradores de rua usuários ou não de álcool e drogas.

Ficou evidente, portanto, que o cenário de vulnerabilidade, somado à ineficácia no diagnóstico, seja pela procura tardia por assistência ou falha no sistema de busca ativa desses pacientes, bem como o desamparo profissional, social e familiar contribuem com a evolução negativa da coinfecção e corroboram com a problemática de saúde pública.

No mais, um dos fatores que ainda permeiam a assistência à saúde são as desigualdades em relação à condição dos pacientes. Por isso, uma percepção justa, humanizada e acolhedora precisa ser praticada entre os profissionais de saúde, para banir os preconceitos que sustentam a exclusão e agravam a saúde, sobretudo, dos marginalizados pela sociedade.

Por fim, esta revisão levantou aspectos importantes que podem conduzir novas investigações em inúmeras particularidades sociais e econômicas, ou seja, análises não generalizadas dos indivíduos nessa condição e, embora, o curto período avaliado, este estudo não perde sua importância, pois denotou problemáticas que são comuns e em sua grande maioria ignoradas e/ou camufladas socialmente, mas que são imprescindíveis no combate, no tratamento ou na prevenção, sobretudo, quando exploradas com outras 
abordagens metodológicas, compreendidas como parte de um processo individualizado e levadas em consideração de modo mais eficaz em políticas de saúde pública.

Afinal, as características socioeconômicas aqui percebidas e relatadas, mostram o quão as disparidades ditam o comportamento dos indivíduos e o quanto essa realidade de nível escolar e de renda baixos e de cor/raça negra ainda persiste, infelizmente, nos estudos de saúde pública, denotando um cenário que perdura há décadas e que pouco se transforma, devido à carência e/ou a ineficácia das ações públicas, fadadas a manutenção de um país altamente desigual e que tem na esfera da saúde suas outras mazelas expostas e, por vezes, esquecidas diante do preconceito estrutural.

\section{Referências}

BATISTA, L. E.; BARROS, S. Enfrentando o racismo nos serviços de saúde. Cadernos de saude publica, v. 33, p. e00090516, 2017.

BRAND, É. M.; ROSSETTO, M.; CALVO, K. D. S.; WINKLER, G. B.; SILVA, D. A. R.; HENTGES, B. et al. Factors associated with directly observed treatment in tuberculosis/HIV coinfection cases in Porto Alegre, 2009-2013: A retrospective cohort. PloS one, v. 14, n. 10, p. e0222786, 2019.

BRASIL. Ministério da Saúde. Secretaria de Vigilância em Saúde. Boletim Epidemiológico: Coinfecção TB-HIV no Brasil: Panorama Epidemiológico e atividades colaborativas 2017. Brasília, DF, 2017a.

BRASIL. Ministério da Saúde. Secretaria de Vigilância em Saúde. Brasil Livre da Tuberculose: Plano Nacional pelo Fim da Tuberculose como Problema de Saúde Pública. Brasília, DF, 2017b.

BRUNELlo, M. E. F.; CHIARAVALlOtI NETO, F.; ARCÊNCIO, R. A.; ANDRADE, R. L. D. P.; MAGNABOSCO, G. T.; VILLA, T. C. S. Áreas de vulnerabilidade para co-infecção HIV-aids/TB em Ribeirão Preto, SP. Revista de saude publica, v. 45, p. 556-563, 2011. 
COSTA, P. A.; ARAÚJO, I. M. C.; BURITI, E. L. S.; MENDONÇA, A. E. O.; ALBUQUERQUE, A. M. CORPOS ESTRANHOS EM PRIMEIROS SOCORROS: REVISÃO INTEGRATIVA. Educação, Ciência e Saúde, v. 7, n. $1,2020$.

DAMÁSIO, G. S.; FRANÇA, H. M.; OLIVEIRA, I. C. M. D.; ARAÚJO, A. R. A. D.; FEIJÃO, A. R. Fatores sociais, clínicos e de adesão em coinfectados por HIV/Tuberculose: estudo descritivo. Online braz. j. nurs.(Online), p. 414-422, 2016.

DANTAS, S. M. V.; COUTO, M. T. Sexualidade e reprodução na Política Nacional de Saúde do Homem: reflexões a partir da perspectiva de gênero. Sexualidad, Salud y Sociedad (Rio de Janeiro), n. 30, p. 99-118, 2018.

ESCADA, R. O. S.; VELASQUE, L.; RIBEIRO, S. R.; CARDOSO, S. W.; MARINS, L. M. S., GRINSZTEJN, E. et al. Mortality in patients with HIV-1 and tuberculosis co-infection in Rio de Janeiro, Brazil-associated factors and causes of death. BMC infectious diseases, v. 17, n. 1, p. 373, 2017.

MAFFACCIOLLI, R.; OLIVEIRA, D. L. L.; BRAND, É. M. Vulnerabilidade e direitos humanos na compreensão de trajetórias de internação por tuberculose. Saúde e Sociedade, v. 26, p. 286-299, 2017.

MAGNABOSCO, G. T.; ANDRADE, R. L. D. P.; ARAKAWA, T.; MONROE, A. A.; VILLA, T. C. S. Desfecho dos casos de tuberculose em pessoas com HIV: subsídios para intervenção. Acta Paulista de Enfermagem, v. 32, n. 5, p. 554563, 2019.

MATOS, T. N. F.; TRAJANO, S. S.; CALDAS, J. M. P.; CATRIB, A. M. F. Representações sociais e condições de saúde em moradores de rua. SANARE-Revista de Políticas Públicas, v. 16, 2017.

MIRANDA, T. N.; TEIXEIRA, J. D. C.; OLIVEIRA, A. C. R.; FERNANDES, R. T. $P$. Fatores que influenciam negativamente na assistência integral ao usuário da atenção básica na saúde do homem. Journal of Health Connections, v. 2, n. 1, 2018. 
NEVES, L. A. S.; CASTRIGHINI, C.; REIS, R. K.; CANINI, S. R. M. S.; GIR, E. Suporte social e qualidade de vida de indivíduos com coinfecção tuberculose/HIV. Enfermería Global, v. 17, n. 2, p. 1-29, 2018.

NOGUEIRA, A. F.; FACCHINETTI, V.; SOUZA, M. V. N. D.; VASCONCELOS, T. R. A. Tuberculose: uma abordagem geral dos principais aspectos. Rev. Bras. Farm, v. 93, n. 1, p. 3-9, 2012.

OLIVEIRA, L. B.; COSTAR, C. R. B.; QUEIROZ, A. A. F. L. N.; ARAÚJO, T. M. E.; SOUSA, K. A. A.; REIS, R. K. ANÁLISE EPIDEMIOLÓGICA DA COINFECÇÃO TUBERCULOSE/HIV. Cogitare Enfermagem, v. 23, n. 1, 2018.

PELISSARI, D. M.; ROCHA, M. S.; BARTHOLOMAY, P.; SANCHEZ, M. N.; DUARTE, E. C.; ARAKAKI-SANCHEZ, D. et al. Identifying socioeconomic, epidemiological and operational scenarios for tuberculosis control in Brazil: an ecological study. BMJ open, v. 8, n. 6, p. e018545, 2018.

PRADO, T. N. D.; RAJAN, J. V.; MIRANDA, A. E.; DIAS, E. D. S.; COSME, L. B.; POSSUELO, L. G. et al. Clinical and epidemiological characteristics associated with unfavorable tuberculosis treatment outcomes in TB-HIV coinfected patients in Brazil: a hierarchical polytomous analysis. Brazilian Journal of Infectious Diseases, v. 21, n. 2, p. 162-170, 2017.

PRADO, T. N.; MIRANDA, A. E.; SOUZA, F. M.; DIAS, E. S.; SOUSA, L. K. F.; ARAKAKI-SANCHEZ, D. et al. Factors associated with tuberculosis by HIV status in the Brazilian national surveillance system: a cross sectional study. BMC infectious diseases, v. 14, n. 1, p. 415, 2014.

RANZANI, O. T.; CARVALHO, C. R.; WALDMAN, E. A.; RODRIGUES, L. C. The impact of being homeless on the unsuccessful outcome of treatment of pulmonary TB in São Paulo State, Brazil. BMC medicine, v. 14, n. 1, p. 41, 2016.

RODRIGUES, J. S.; DE LIMA, A. F.; HOLANDA, R. B. Identidade, drogas e saúde mental: narrativas de pessoas em situação de rua. Psicologia: Ciência e Profissão, v. 38, n. 3, p. 424-436, 2018. 
ROSSETTO, M.; MAFFACCIOLLI, R.; ROCHA, C. M. F.; OLIVEIRA, D. L. L. C. D.; SERRANT, L. Coinfecção tuberculose/HIV/aids em Porto Alegre, RSinvisibilidade e silenciamento dos grupos mais afetados. Revista Gaúcha de Enfermagem, v. 40, 2019.

SANTOS, D. T. D.; GARCIA, M. C.; COSTA, A. A. N. F. D.; PIERI, F. M.; MEIER, D. A. P.; ALBANESE, S. P. R. et al. Infecção latente por tuberculose entre pessoas com HIV/AIDS, fatores associados e progressão para doença ativa em município no Sul do Brasil. Cadernos de Saúde Pública, v. 33, p. e00050916, 2017.

SILVA, A. N. M.; SOUSA, M. N. A.; LIMA, A. B.; SILVA, M. C. L.; CAMBOIM, J. C. A.; CAMBOIM, F. E. F. Vivências de portadores de tuberculose e importância da família à adesão terapêutica. Interfaces Científicas-Saúde e Ambiente, v. 6, n. 1, p. 83-94, 2017.

SILVA, J. B.; CARDOSO, G. C. P.; NETTO, A. R.; KRITSKI, A. L. Os significados da comorbidade para os pacientes vivendo com TB/HIV: repercussões no tratamento. Physis: Revista de Saúde Coletiva, v. 25, p. 209-229, 2015.

SOUZA, M. T.; SILVA, M. D.; CARVALHO, R. Revisão integrativa: o que é e como fazer. Einstein (São Paulo), v. 8, n. 1, p. 102-106, 2010.

WILLIAMS, D. R.; PRIEST, N. Racismo e Saúde: um corpus crescente de evidência internacional. Sociologias, v. 17, n. 40, 2015.

WORLD HEALTH ORGANIZATION. Global Tuberculosis Report 2017. Geneva, Switzerland, 2017. 


\section{Agradecimentos}

À Liga Acadêmica de Anatohistofisiologia, da Universidade do Estado do Pará, campus XII, por proporcionar aos seus ligantes, o incentivo à produção científica. 
EDUCAÇÃO CIÊNCIA E SAÚDE | http://dx.doi.org/10.20438/ecs.v7i2.294 